\title{
COMPLICACIONES DEL USO DE CATÉTER VENOSO CENTRAL EN PACIENTES DE LA UNIDAD DE CUIDADOS INTENSIVOS EN EL HOSPITAL GENERAL PLAZA DE LA SALUD EN EL PERÍODO DE JUNIO 2017 - JUNIO 2018
}

\author{
Complications of the use of central venous catheter in patients of \\ the Intensive Care Unit at the General Hospital Plaza de la Salud \\ in the period from June 2017 - June 2018
}

\section{Darlene Espiritusanto Loraa , Jasmín Cabrera Luis ${ }^{b}$, Emilia Eunice Cid ${ }^{c}$}

Recibido: 2 de junio, 2019 • Aprobado: 1 de septiembre, 2019

Cómo citar: Espiritusanto Lora D, Cabrera Luis J, Eunice Cid E. Complicaciones del uso de catéter venoso central en pacientes de la Unidad de Cuidados Intensivos en el Hospital General Plaza de la Salud en el período de junio 2017 - junio 2018 . cysa [Internet]. 16 de junio de 2020 [citado 17 de junio de 2020];4(2):71-79. Disponible en: https://revistas.intec.edu.do/index.php/cisa/article/ view/1761

\section{Resumen}

Introducción: el uso de un catéter venoso central es esencial para el manejo de miles de paciente en los centros hospitalarios en República Dominicana y el resto del mundo. No obstante, los CVC están asociados a varias complicaciones, incluyendo algunas que son potencialmente fatales.

Método: se realizó un estudio observacional descriptivo retrospectivo en el área de UCI en el Hospital General de la Plaza de la Salud, en el cual se evaluaron los historiales clínicos de 93 pacientes a los que se les colocó un CVC en el periodo de junio 2017 - junio 2018.

Conclusión: en este estudio se observó que dentro de una muestra de 93 pacientes a los cuales se les colocó un CVC en el Hospital General de la Plaza de la Salud en el periodo junio 2017- junio 2018, un $19 \%$ presentaron complicaciones por CVC, siendo la infección la complicación más frecuente.

Palabras clave: catéter venoso central; infección; oclusión; extravasación; hematoma.

\footnotetext{
a Doctora en Medicina, Hospital Central de las Fuerzas Armadas (HCFFAA), República Dominicana. ORCID: 0000-0002-6250-4357 Correo-e: darleneel299@gmail.com
}

\begin{abstract}
Introduction: In the Dominican Republic the use of a central venous catheter is essential for the management of thousands of patients in hospital centers. However, CVCs are associated with several complications, including some that are potentially fatal.

Method: A retrospective, descriptive, observational study was conducted in the ICU at the General Hospital of the Plaza de la Salud in which the clinical records of 93 patients who were placed on a CVC in the period of June 2017-June 2018 were evaluated.

Conclusion: In this study, it was observed that within a sample of 93 patients to whom a CVC was placed in ICU of the General Hospital of the Plaza de la Salud in the period June 2017- June 2018, 19\% presented complications due to $\mathrm{CVC}$, being the infection the most frequent complication.
\end{abstract}

Keywords: Central venous catheter; infection; occlusion; extravasation; hematoma.

\footnotetext{
b Doctora en Medicina, Hospital Central de las Fuerzas Armadas (HCFFAA), República Dominicana. ORCID: 0000-0002-8007-2359 Correo: jasminncabreraluis@hotmail.com

c Doctora en Medicina, Cardióloga Intervencionista, Hospital General Plaza de la Salud (HGPS), República Dominicana.

Correo: eunice-cid@hotmail.com
} 


\section{Introducción}

La cateterización venosa se define como la inserción de un catéter biocompatible en el espacio intravascular, central o periférico, con fines diagnósticos y/o terapéuticos. La canalización venosa central (CVC) es una técnica cuyo empleo está creciendo progresivamente a nivel hospitalario debido al aumento de la edad, comorbilidad y gravedad de los pacientes hospitalizados. Algunas de las indicaciones de la utilización de catéter venoso central incluyen nutrición parenteral total (NPT), administración de fármacos, antibioterapia a largo plazo, quimioterapia, drogas vaso activas, hemodiálisis, monitorización hemodinámica o imposibilidad de acceso venoso periférico. ${ }^{1}$

En ocasiones no es posible la cateterización venosa a través de las vías habituales de acceso, debido a la inestabilidad hemodinámica, respiratoria y/o neurológica del paciente, o bien porque catéteres previos han favorecido la formación de zonas fibróticas que dificultan la introducción de los mismo. El acceso venoso central en el paciente depende de la experiencia y entrenamiento del intensivista y/o cirujano, así como también de la edad, peso, presencia de patologías cervicales, condición anatómica y alteraciones respiratorias del paciente. ${ }^{1}$

Desafortunadamente, cuando un paciente con catéter venoso central se complica, se asocia con un aumento de la estancia hospitalaria y se elevan los costos médicos. Para mejorar los resultados clínicos y disminuir los costos sanitarios, se deben diseñar estrategias con el objetivo de reducir la incidencia de estas complicaciones. Se estima que aproximadamente entre un 40 y un $46 \%$ de los catéteres venosos centrales tanto en niños como en adultos pueden desarrollar complicaciones. ${ }^{1}$

Teniendo en cuenta lo anterior, es imperativo planificar, desde el principio del tratamiento, las nece- sidades de accesos venosos centrales para reducir al mínimo la cantidad de punciones venosas a un paciente crítico. ${ }^{1}$

No obstante, los CVC están asociados a varias complicaciones, incluyendo algunas que son potencialmente fatales (por ejemplo, neumotórax y sepsis asociada al catéter) ${ }^{2}$. Son miles los pacientes ingresados en las UCI de los hospitales portadores de CVC. En EE. UU. se producen 80,000 bacteriemias en pacientes portadores de catéteres centrales de los que se derivan 28,000 muertes cada año. La CDC reporta una tasa de mortalidad de 12 a $15 \%$ cuando una infección de vía central se vuelve sistémica. ${ }^{3}$

\section{Material y métodos}

Este estudio es de tipo observacional descriptivo con datos retrospectivos, que buscó determinar las complicaciones de la colocación de un catéter venoso central en pacientes adultos ingresados en la unidad de cuidados intensivos del Hospital General Plaza de la Salud, a los que se colocó catéter venoso central durante el periodo comprendido de junio del 2017 a junio del 2018. En esta investigación se describió el problema y se completaron los objetivos, tales como determinar la prevalencia de complicaciones tras la colocación de un catéter venoso central en pacientes adultos ingresados en la unidad de cuidados intensivos. El estudio es de tipo retrospectivo, ya que se llevó a cabo analizando expedientes de pacientes que hayan sido ingresados por la unidad de cuidados intensivos y que hayan requerido un catéter venoso central.

La recolección de datos se realizó utilizando una ficha de observación que se completó con el uso de los expedientes de los pacientes con complicaciones asociadas a CVC en la UCI en el Hospital General de la Plaza de la Salud del 2017-2018. La ficha de observación se desarrolló utilizando los determinados indicadores de las variables. 
La población incluye a todos los adultos ingresados en la unidad de cuidados intensivos en el Hospital General Plaza de la Salud, en el periodo comprendido entre julio del 2017 a julio del 2018, de los cuales requirieron la colocación de catéter venoso central.

\section{Criterios de inclusión}

1. Pacientes adultos que ingresaron a unidad de cuidados intensivos, que se les colocó catéter venoso central.

2. Pacientes adultos que ingresaron a cuidados intensivos sin habérsele colocado catéter en otra unidad de salud.

\section{Criterios de exclusión}

1. Expedientes clínicos extraviados.

2. Expedientes clínicos con datos incompletos.

3. Pacientes que fueron trasladados a otra unidad y se desconoce seguimiento del catéter venoso central.

En esta investigación se utilizó una ficha de recolección de datos para organizar las variables y sus indicadores. La ficha se completó utilizando los expedientes clínicos de los pacientes con complicaciones asociadas a CVC en la UCI en el Hospital General de la Plaza de la Salud del 2017-2018. Los programas tecnológicos que se utilizaron fueron Microsoft Office Excel y Epi Info ${ }^{\text {тм }} 2018$.

Se respetaron los datos personales de los pacientes seleccionados para esta investigación, garantizando la confidencialidad y no divulgación de ningún antecedente y dato personal de cada uno de los pacientes. Nuestra investigación fue aprobada por el comité de ética de la Universidad Iberoamericana y la Dirección del Hospital General Plaza de la Salud.

\section{Resultados y discusiones}

Gráfica 1. Género de la muestra entrevistada, junio 2017- junio 2018. $\mathrm{N}=93$.

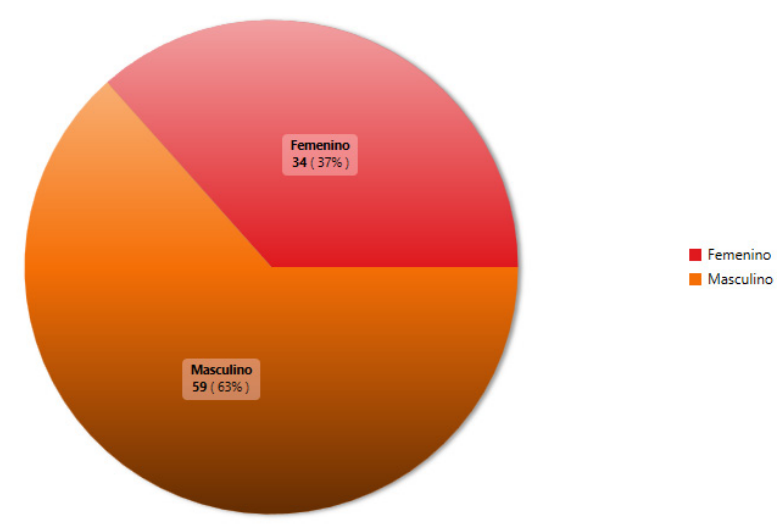

Fuente: elaboración propia. Junio 2017- junio 2018.

Este gráfico muestra que dentro del estudio se contó con un total de 93 pacientes de los cuales, un $63 \%$ era de sexo masculino, y un $37 \%$ del sexo femenino.

Gráfica 2. Rango etario de la muestra estudiada, junio 2017- junio 2018. $\mathrm{N}=93$.

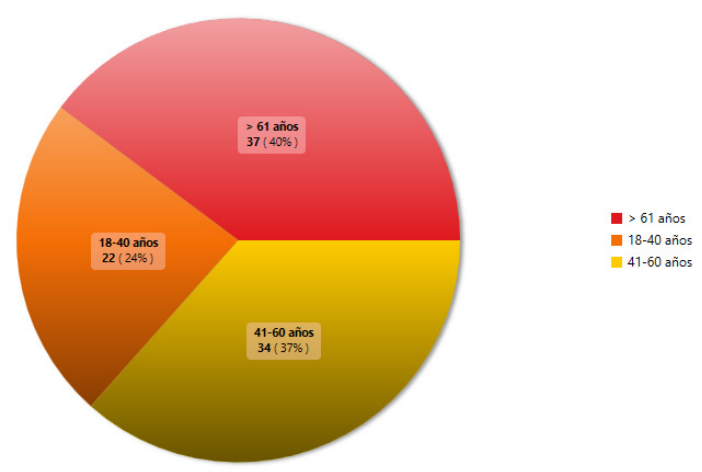

Fuente: elaboración propia. Junio 2017- junio 2018.

Dentro de los rangos de edad observamos que un $40 \%$ de los pacientes con CVC eran mayor de 60 años, un $37 \%$ se encontraba dentro de las edades 41 a 60 ańos, y el restante, $24 \%$, en el rango de 18 a 40 años de edad. 
Gráfica 3. Cantidad de pacientes que presentaron una complicación asociada a la colocación de catéter venoso central, junio 2017- junio 2018. $\mathrm{N}=93$

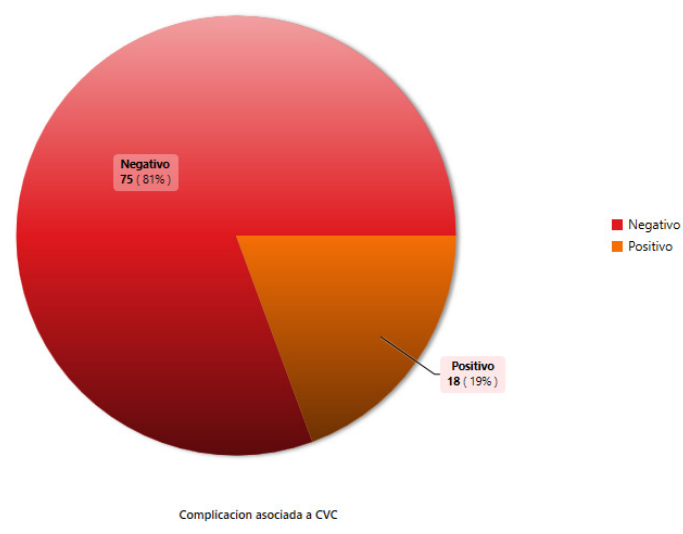

Fuente: elaboración propia. Junio 2017- junio 2018.

En esta gráfica representamos el porcentaje de pacientes que presentaron una complicación directamente asociada al catéter venoso central. Podemos ver que un $19 \%$ de los pacientes presentaron alguna complicación, mientras que el restante, $81 \%$, no presentó complicación asociada al CVC.
Gráfica 4. Complicaciones más frecuentes que surgieron tras la colocación de catéter venoso central, junio 2017- junio 2018. $\mathrm{N}=93$

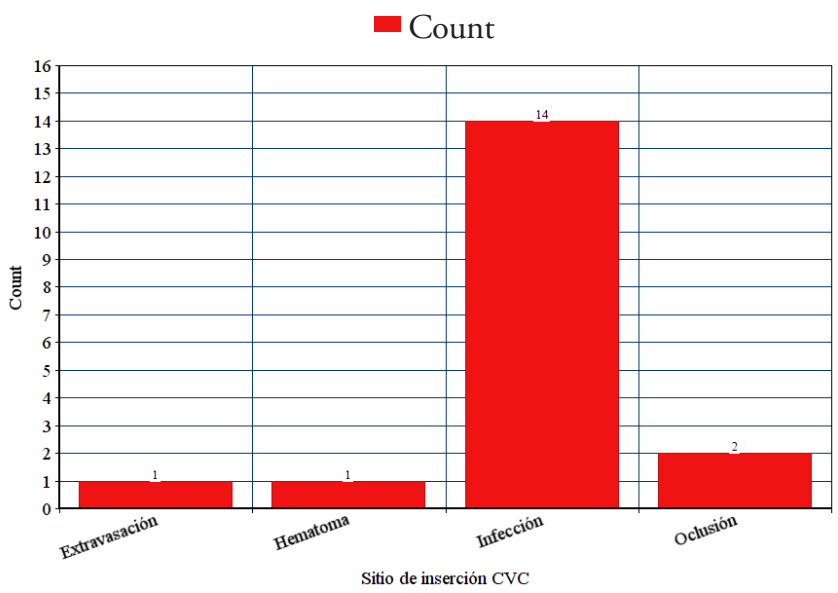

Fuente: elaboración propia. Junio 2017- junio 2018.

En esta gráfica se evidencian las complicaciones más frecuentes tras la colocación del CVC. Vemos que el mayor porcentaje de los pacientes presentaron infección por contaminación del catéter, con un $77 \%$.

Gráfica 5. Tipo de complicación más frecuentes de acuerdo con el tiempo en días que el paciente permaneció con la canalización venosa central, junio 2017- junio 2018. N = 93
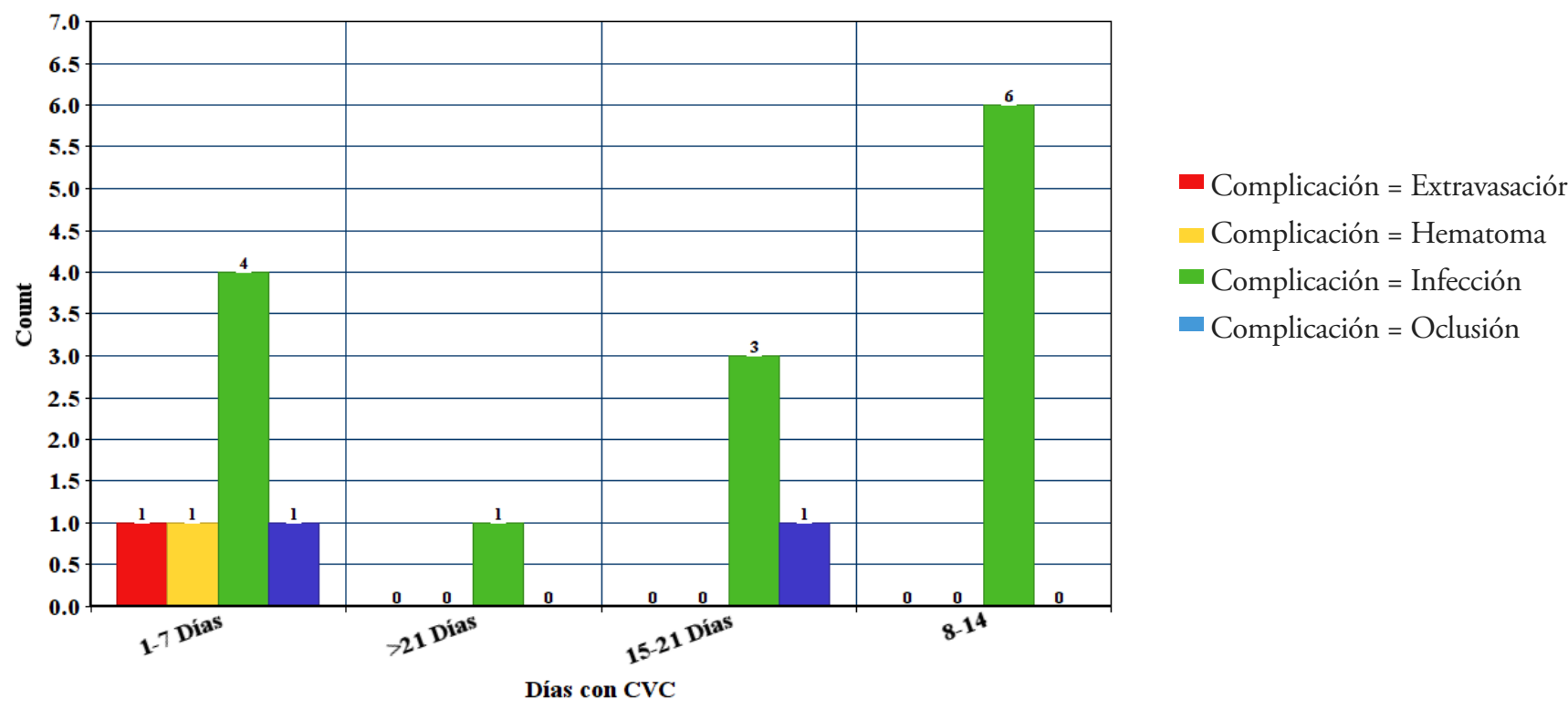

Fuente: elaboración propia. Junio 2017- junio 2018. 
Dentro de los pacientes que permanecieron 1-7 días con la vía central, encontramos la mayor cantidad de complicaciones mecánicas, mientras que el mayor porcentaje de pacientes con complicaciones infecciosas $(43 \%)$ permanecieron de 8 -14 días con el CVC.

Gráfica 6. Grado de correlación entre el diagnóstico de ingreso del paciente a UCI y el tipo de complicación presentada, junio 2017- junio 2018. $\mathrm{N}=93$

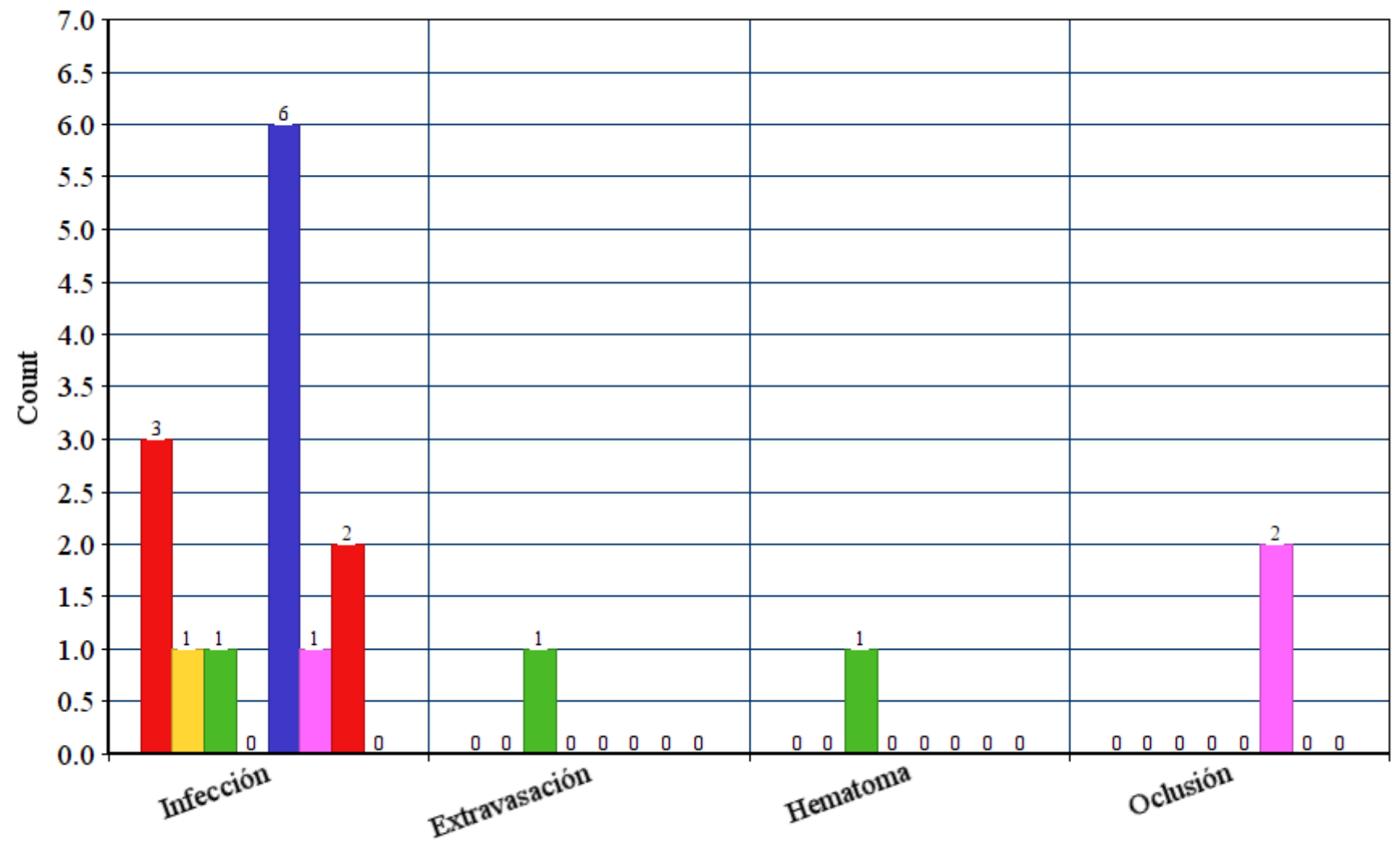

Complicación

- Diagnóstico de ingreso = Cardiovascular

Diagnóstico de ingreso $=$ Endocrino

- Diagnóstico de ingreso = Gastrointestinal

- Diagnóstico de ingreso = Genitourinario
- Diagnóstico de ingreso $=$ Nervioso

- Diagnóstico de ingreso = Otro

- Diagnóstico de ingreso $=$ Respiratorio

- Diagnóstico de ingreso = Trauma

Fuente: elaboración propia. Junio 2017- junio 2018. 
Analizamos la correspondencia entre el diagnóstico de ingreso del paciente a UCI y la complicación presentada. Dentro de los pacientes que presentaron infección como complicación, un $43 \%$ tuvo un diagnóstico de ingreso del sistema nervioso central, seguido por un $21 \%$ con un diagnóstico de ingreso del sistema cardiovascular y un $14 \%$ del sistema respiratorio.

Gráfica 7. Grado de morbilidad de las complicaciones de colocación de CVC de acuerdo con el lugar anatómico de dicha colocación, junio 2017 - junio 2018. N = 93

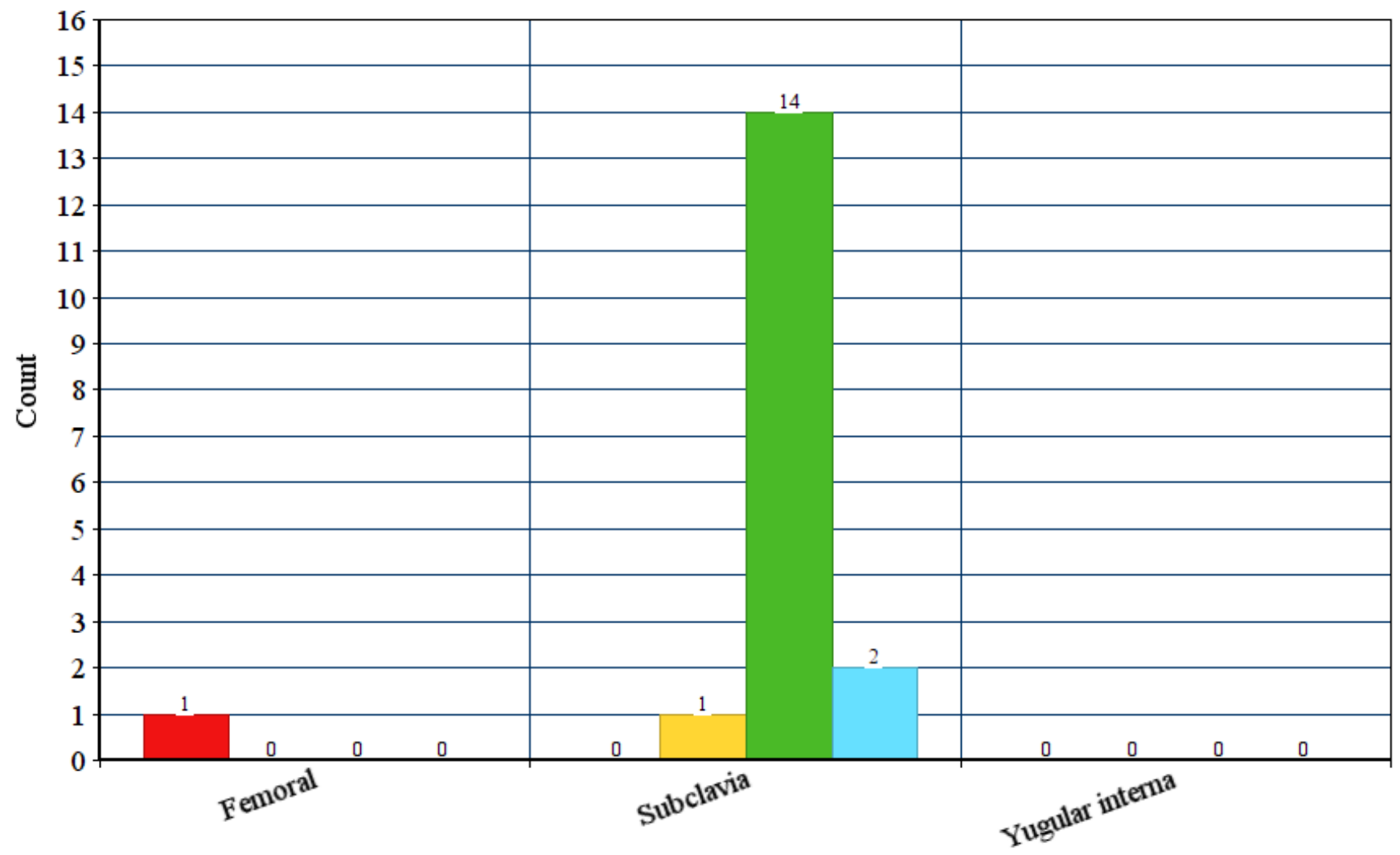

Sitio de inserción CVC

- Complicación = Extravasaciór

- Complicación = Hematoma
- Complicación = Infección

- Complicación = Oclusión

Fuente: elaboración propia. Junio 2017- junio 2018.

Comparamos el tipo de complicación secundaria a CVC con el sitio anatómico de dicha colocación. Dentro del $19 \%$ de pacientes que presentó compli- caciones, un $94 \%$ tuvo como sitio de inserción del CVC la vena subclavia, siendo la infección la complicación más común en esta. 
Gráfica 8. Diagnóstico de egreso de los pacientes con colocación de catéter venoso central en la Unidad de Cuidados Intensivos, junio 2017 - junio 2018. $\mathrm{N}=93$.

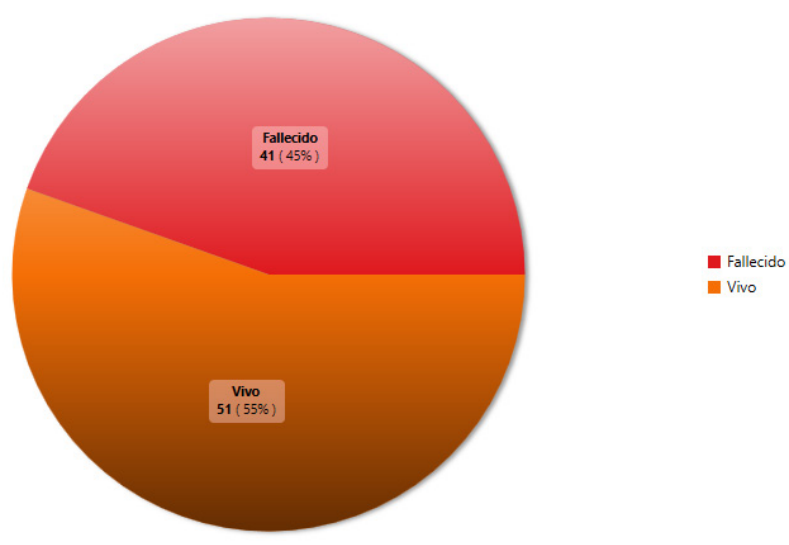

Fuente: elaboración propia. Junio 2017- junio 2018.

Aquí vemos el diagnóstico de egreso de los pacientes fueron traslados a sala, mientras que un $45 \%$ fueron con colocación de catéter venoso central en pacientes defunciones. de la Unidad de Cuidados Intensivos. Un $55 \%$

Gráfica 9. Relación entre las complicaciones por colocación de CVC y las defunciones en la Unidad de Cuidados Intensivos, junio 2017 - junio 2018.

$\mathrm{N}=93$

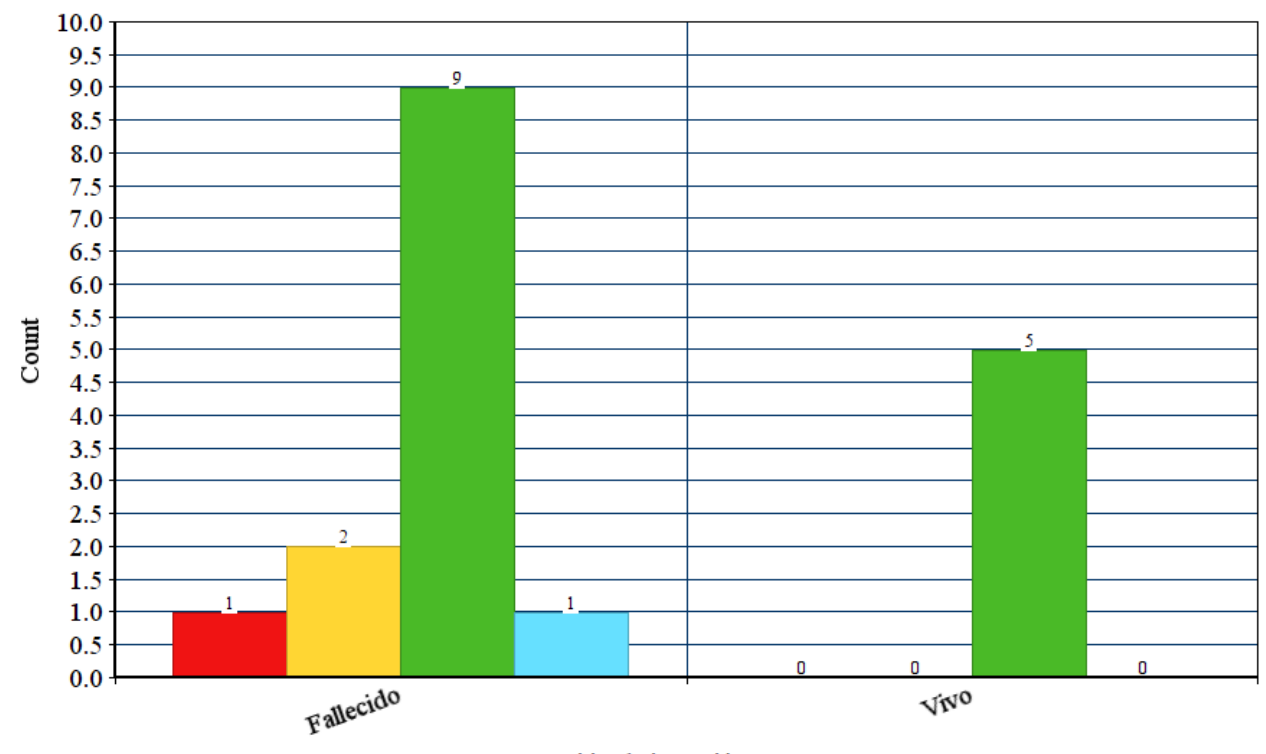

- Complicación = Extravasaciór

Complicación = Hematoma

- Complicación = Infección

- Complicación = Oclusión

Fuente: elaboración propia. Junio 2017 - junio 2018. 
Exploramos sobre la posible existencia de una relación entre las complicaciones por colocación de CVC y las defunciones, donde pudimos ver que CVC, un $72 \%$ fallecieron presentando infección como complicación secundaria a CVC. El restante, $28 \%$ de los pacientes, todos complicados con infección (36\%), fueron dados de alta. A pesar de esto, concluimos de acuerdo a la información que adquirimos en los historiales, que las complicaciones por CVC no fueron causas directas de estas defunciones, sino que la mayoría de estos pacientes sucumbieron a fallo multi-orgánico asociado a su diagnóstico de ingreso a UCI.

\section{Conclusiones}

Pudimos observar que un $19 \%$ evidenció complicaciones, mientras que el restante $81 \%$ no presentó ninguna complicación asociada al catéter venoso central.

Dentro del grupo que exhibió complicaciones, un $77 \%$ presentó infección por contaminación del catéter, $11 \%$ presentó oclusión, $6 \%$ hematoma y $6 \%$ extravasación, evidenciando la infección como complicación más común del uso de catéter venoso central en este estudio, lo cual está acorde con la literatura ${ }^{10}$ que establece la sepsis como complicación más común secundaria a la contaminación del catéter venoso central. En el estudio se consideró información sobre el tiempo en días que el paciente permaneció con la canalización venosa central. Dentro de los pacientes que permanecieron 1-7 días con la vía central, encontramos la mayor cantidad de complicaciones mecánicas, mientras que el mayor porcentaje de pacientes con complicaciones infecciosas $(43 \%)$ permanecieron de 8-14 días con el CVC, lo cual es congruente con la literatura Schwartz ${ }^{10}$ que plantea que una duración mayor de 7 días con un CVC está asociada a un incremento de $5 \%$ a $10 \%$ de riesgo a infección del catéter. Se analizó la correspondencia entre el diagnóstico de ingreso del paciente a UCI y la complicación presentando por el CVC.
De igual manera comparamos el tipo de complicación secundaria a CVC con el sitio anatómico de dicha colocación. Dentro del $19 \%$ que demostró complicaciones, un $94 \%$ tuvo como sitio de inserción del CVC la vena subclavia, siendo la infección la complicación más común $(82 \%)$ en esta. La inserción del CVC en la vena femoral exhibió un $6 \%$ complicado con extravasación, mientras que los pacientes con inserción de CVC en la vena yugular interna no presentaron complicación. En la mayoría de nuestros pacientes se eligió la vena subclavia como lugar anatómico para la inserción de CVC. En la literatura, el riesgo de infección como una complicación de la inserción de CVC es menor en la vena subclavia, seguida por la yugular y siendo la femoral la de mayor riesgo ${ }^{10}$. Consideramos que el alto porcentaje de pacientes referidos con una infección como complicación de la inserción de un CVC en la vena subclavia se debe a que este fue el lugar anatómico que se utilizó con mayor frecuencia.

\section{Bibliografía}

1. Davis M. Pediatric Central Venous Catheter Management: A Review of Current Practice. Journal of the Association for Vascular Access [Internet]. 2013;18(2):93-8. Available from: https://www.sciencedirect.com/science/article/ abs/pii/S1552885513000433

2. Davis M. Pediatric Central Venous Catheter Management: A Review of Current Practice. Journal of the Association for Vascular Access [Internet]. 2013;18(2):93-8. Available from: https://www.sciencedirect.com/science/article/ abs/pii/S1552885513000433

3. OMS | Prevención de las Bacteriemias relacionadas con Catéteres Venosos Centrales (BRC) en las UCI Españolas. [Internet]. Who.int. 2020 [cited 2 March 2019]. Available from: https:// www.who.int/patientsafety/activities/bacteriemia_zero/es/ 
4. McPheeters R, Catoera I. Central Venous Access Via External Jugular Vein: Background, Indications, Contraindications [Internet]. Emedicine. medscape.com. 2019. Available from: https:// emedicine.medscape.com/article/2116323-overview [cited 4 March 2019]

5. Sznajder J. Central Vein Catheterization. Archives of Internal Medicine [Internet]. 1986;146(2):259. Available from: https://jamanetwork.com/journals/jamainternalmedicine/ article-abstract/606564

6. Mirski M. Diagnosis and Treatment of Vascular Air Embolism. Survey of Anesthesiology [Internet]. 2007;51(3):157. Available from: https://anesthesiology.pubs.asahq.org/article. aspx?articleid $=1923071$

7. Ruesch S, Walder B, Tramèr M. Complications of central venous catheters: Internal jugular versus subclavian access - A systematic review. Critical Care Medicine [Internet]. 2002; 30(2):454-60. Available from: https://www.ncbi.nlm.nih.gov/ books/NBK69121/

8. Eisen L, Narasimhan M, Berger J, Mayo P, Rosen M, Schneider R. Mechanical Complications of Central Venous Catheters. Journal of Intensive Care Medicine [Internet]. 2006;21(1):40-6. Available from: https://www.ncbi.nlm.nih.gov/ pubmed/16698743

9. Shah K, Rao T, Laughlin S, El-Etr A. A Review of Pulmonary Artery Catheterization in 6,245 Patients. Anesthesiology [Internet]. 1984;61(3): 271-5. Available from: https://europepmc.org/ article/med/6476435

10. Brunicardi F, Anderson D. Schwartz's principles of surgery. 10th ed. New York: McGraw-Hill; 2015.
11. Allen-Bridson K. NHSN Central Line-associated Bloodstream Infection Surveillance in 2014 [Internet]. CDC.gov. 2014. Available from: https://www.cdc.gov/nhsn/PDFs/training/ training-CLABSI-2014-with-answers-BW.pdf [cited 10 March 2019]

12. Dumont C, Nesselrodt D. Preventing central line-associated bloodstream infections CLABSI. Nursing [Internet]. 2012;42(6):41-6. Available from: https://journals.lww.com/nursing/FullText/2012/06000/Preventing_central_line_ associated_bloodstream.14.aspx

13. Cárcoba Rubio N, Ceña Santorcuato S. Cateterización venosa central de acceso periférico mediante técnica seldinger modificada en la urgencia hospitalaria. Enfermería Global [Internet]. 2010;(20). Available from: http:// scielo.isciii.es/scielo.php?script=sci_arttext\&pid=S1695-61412010000300015 [cited 16 March 2019]

14. Central Venous Catheterization. New England Journal of Medicine [Internet]. 2007;357(9): 943-5. Available from: https://www.nejm. org/doi/full/10.1056/NEJMc071785 [cited 10 March 2019]

15. Urrea Ayala M, Rozas Quesada L. La bacteriemia asociada al catéter venoso central: implementación de un nuevo protocolo de consenso. Anales de Pediatría [Internet]. 2009;71(1):20-4. Available from: https://www.sciencedirect.com/science/article/abs/pii/S1695403309002537 [cited 17 March 2019] 